\title{
El periodismo y la necesidad de estar bien informado. Una propuesta formativa
}

\author{
Simón PeÑa FernándeZ \\ Universidad del País Vasco \\ simon.pena@ehu.es \\ Iñaki LAZKANO ARRILLAGA \\ Universidad del País Vasco \\ Antxoka Agirre Maiora ${ }^{1}$ \\ Universidad del País Vasco
}

\section{Resumen:}

El desarrollo del hábito de estar bien informado es una competencia de vital importancia en la formación de los futuros periodistas y comunicadores. Este artículo describe, en el marco de un Proyecto de Innovación Educativa, el desarrollo de la plataforma gaurtest.info con la que los alumnos de los grados del área de Comunicación pueden autoevaluar su conocimiento en este área mediante la elaboración de test de actualidad online.

Palabras clave: Actualidad informativa; Innovación educativa; Test online; Autoevalución de competencias; Comunicación; Periodismo

\section{Journalism and the Need of Being Well Informed. A Training Proposal.}

\begin{abstract}
:
Developing the habit of being well informed is a key ability in future journalists and communicators training. This article describes, in the context of a teaching innovation project, the creation and use of the gaurtest.info platform. In this website, undergraduate students from Communication degrees can self assess their knowledge in this area by answering to online tests about current news.
\end{abstract}

Key Words: Current News; Teaching Innovation; Online Test; Self Assess of Abities; Communication; Journalism

\section{Referencia normalizada:}

Peña Fernández, S., Lazkano Arrillaga, I. y Agirre Majora, A. (2014): El periodismo y la necesidad de estar bien informado. Una propuesta formativa. Historia y Comunicación Social. Vol. 19. Núm. Especial Febrero. Págs. 387-400.

Sumario: 1. Introducción. 2. Metodología. 3. La plataforma gaurtest.info. 4. Resultados del aprendizaje. 5. Conclusiones.

1 Los autores quieren agradecer al Servicio de Asesoramiento Educativo y al Vicerrectorado de Calidad e Innovación Docente de la Universidad del País Vasco (UPV/EHU) la financiación recibida para desarrollar este proyecto. Asimismo, quieren reconocer la labor de las profesoras María José Cantalapiedra y Leire Iturregui, quienes también han trabajado activamente en el desarrollo de esta investigación. Igualmente, los autores desean agradecer la colaboración de los 413 alumnos y alumnas que han participado en este proyecto. 


\section{Introducción}

Los comunicadores, y en particular quienes desarrollan labores periodísticas en los medios de comunicación, están obligados a trabajar de forma permanente con grandes volúmenes de datos e información que no sólo deben analizar y seleccionar, sino también elaborar. Comunicar los acontecimientos más recientes alude directamente a la esencia misma de la labor periodística que, tal y como la define Ángel Benito Jaén, puede entenderse como "la selección de aquellos acontecimientos que, en razón de su interés humano y actual, ganan la atención pública en un momento dado" (Benito Jaén, 1995. Pág. 11).

Por su parte, Mar de Fontcuberta subraya el hecho de que la actualidad es el factor que convierte un hecho en digno de ser noticia y, por tanto, es el eje de la actividad periodística.

A pesar de que la actualidad inmediata sigue siendo una de las razones de ser básicas del periodismo, cada vez aumenta la coexistencia en los medios de hechos que, más que explicar la ruptura o las incidencias de una normalidad, muestran el desarrollo de la cotidianidad. En ese sentido, los medios y en particular la prensa escrita, se dedican a analizar procesos y tendencias sociales cuya actualidad se amplía a períodos largos que ocupan meses, años o décadas (De Fontcuberta, 1993. Pág. 25).

Para desarrollar esta tarea informativa resulta esencial comprender que esta materia prima sobre la que se trabaja en los medios de comunicación no está conformada por sucesos inconexos entre sí, sino que todas ellos se enmarcan en complejos procesos que producen muchas unidades similares a lo largo del año. Y esta necesaria contextualización, unida a la obligación de informar de forma veraz y eficiente -indispensable para el correcto ejercicio de la función social de los periodistas y comunicadores- requiere de profesionales que hayan desarrollado el hábito de estar debidamente informados.

El obligado conocimiento de los antecedentes, las causas y las posibles consecuencias de los acontecimientos o, lo que es lo mismo, la capacidad de contextualizar los sucesos, se convierte así en un requisito indispensable en la cotidiana labor de intermediación entre la realidad y los lectores. Quien quiera informar sobre política internacional o economía debe conocer la materia sobre la que trabaja con la misma dedicación que el médico especialista conoce la suya. Y en un entorno en el que el exceso de informaciones resulta cada vez más evidente, resulta más indispensable que nunca afianzar la determinación y el criterio con el que se aborda esta tarea (Peña et al., 2011. Pág. 65).

Puede concluirse, por tanto, que el objeto de estudio es en realidad una materia cambiante, inscrita en largos procesos temporales. $\mathrm{Y}$ esta circunstancia conduce necesariamente a la indispensable reflexión sobre la relevancia y necesidad que tiene el desarrollo del hábito de estar debidamente informado en la formación de los profesionales, y el modo en el que éste se puede desarrollar desde las aulas universitarias. 
La experiencia obtenida en esta tarea, por el contrario, ha revelado de forma recurrente una paradoja en los hábitos de consumo de información percibidos en los futuros comunicadores y periodistas que pueblan las aulas universitarias. Las pruebas de actualidad que se realizan de forma periódica en las materias de redacción periodística de primer curso de las titulaciones de Comunicación (Comunicación Audiovisual, Periodismo y Publicidad y Relaciones Públicas) de la Facultad de Ciencias Sociales y de la Comunicación de la UPV/EHU revelan, de forma mayoritaria, unos hábitos de consumo de informaciones muy poco desarrollados y un conocimiento epidérmico de la actualidad informativa, cuando no un desinterés manifiesto por conocer cuanto acontece en la realidad circundante.

Incrementar el consumo de informaciones por parte del alumnado y su conocimiento de la actualidad como parte de su proceso de formación como comunicadores y periodistas se presenta, en este contexto, como una necesidad básica para los futuros profesionales. Y estar informado no puede enseñarse como si de un tema más se tratara, sino que debe inculcarse en el alumno como un hábito. Los estudiantes de Comunicación deben comprender que para el desarrollo de su profesión resulta fundamental, además del bagaje de conocimientos adquiridos durante la carrera, estar permanentemente informados sobre su área de trabajo.

Más aún, y tal y como apuntaba el reportero polaco Ryszard Kapuscinski, esta indispensable contextualización incrementa la seguridad de quien comunica y le ayuda además a desarrollar una buena escritura.

Si vamos a hablar de fenómenos sociales, por ejemplo, debemos construir el enfoque de una manera amplia: la filosofía, la antropología, la psicología de ese fenómeno. No podemos adentrarnos en el campo social y político sin antes leer mucho; eso es indispensable no sólo para no caer en descubrimientos hechos por otros, sino porque la lectura previa da fuerza a nuestra prosa. Si un autor se siente inseguro acerca del objeto de su trabajo, inmediatamente su escritura deja ver esa falta de confianza. La fuerza de la prosa viene de nuestra seguridad (Kapuscinski, 2003. Pág. 42).

Dentro del ámbito de la formación superior, los planes de estudios y los programas de las materias han incorporado paulatinamente la preocupación por el desarrollo de este hábito. En el caso de las titulaciones del área de Comunicación impartidas en la Facultad de Ciencias Sociales y de la Comunicación la UPV/EHU, entre sus competencias de titulación se recoge que el alumno debe ser capaz de "analizar, interpretar y explicar los hechos y procesos sociales a partir de los conocimientos y metodologías propios de las ciencias sociales en el contexto de la labor de los medios de comunicación". Por su parte, la materia de primer curso Géneros Informativos, obligatoria en los tres grados, recoge entre sus competencias específicas la exigencia del conocimiento de la actualidad informativa por parte del alumnado.

Por este motivo, las asignaturas de redacción periodística han incorporado como recurso habitual la realización de test de actualidad, un recurso que en gran medida el ha sido también el más característico. El número de horas presenciales en el aula, sin embargo, ha limitado tradicionalmente el tiempo que podía dedicarse a esta acti- 
vidad, siempre en pugna con los contenidos de las clases magistrales y las prácticas que debe realizar el alumno.

Para ello, y con el objetivo de diseñar y desarrollar pruebas de actualidad online que pudieran servir como instrumento de aprendizaje y autoevaluación en la materia de redacción periodística Géneros informativos, se diseñó y puso en marcha durante el periodo 2010-2012 el Proyecto de Innovación Educativa titulado 'Los test de actualidad online como herramienta autoformativa para la adquisición de competencias en el área de comunicación'. Este proyecto, financiado por el Vicerrectorado de Calidad e Innovación Docente de la UPV/EHU, se enmarca asimismo en el desarrollo del modelo de enseñanza-aprendizaje cooperativo y dinámico denominado IKD, en el que se impulsa el aprendizaje autónomo del alumnado -centro del proceso educativo- mediante el uso de metodologías activas, en un contexto plurilingüe y aprovechando el uso de las tecnologías de la información.

En este propósito, el empleo de las nuevas tecnologías resulta de gran ayuda para los docentes, no sólo en su aspecto técnico, ni por la transformación que provocan en las metodologías empleadas, sino también por el énfasis que permite poner en la autonomía y la participación del alumnado (Palomares et al., 2005. Pág. 145). Así, a pesar de que el profesorado trace y sugiera en el aula las líneas maestras de esta tarea, e incluso realice puntualmente pruebas de evaluación para conocer la progresión del alumnado, el desarrollo fundamental de la actividad tendrá por fuerza un carácter no presencial. No es suficiente recordar la importancia de realizar un consumo habitual de la información periodística que ofrecen los diversos medios de comunicación, sino que resulta más eficaz proporcionar al alumnado herramientas para que evalúe su competencia y su progresión, siempre acompañado de pautas que les ayuden a profundizar en aquellos aspectos en los que su conocimiento es menor.

\section{Metodología}

El diseño de una herramienta online que contribuyera al desarrollo del hábito de estar informado partió de tres premisas iniciales, la primera de las cuales contemplaba que el alumnado tuviera la posibilidad de trabajar de forma autónoma en las horas de aprendizaje no presencial. En este propósito, el desarrollo de una herramienta virtual permite, tal y como señalan García-Beltrán et al., realizar un seguimiento individualizado del aprendizaje y evaluar sus conocimientos, favorece su flexibilidad temporal y espacial, y resulta especialmente útil para que cada alumno pueda seguir su propio ritmo, bien obteniendo los resultados de manera inmediata o bien almacenándolos para elaborar informes (García-Beltrán et al., 2006. Pág. 4). Asimismo, esta herramienta online debía permitir, en segundo lugar, que los estudiantes autoevaluaran su conocimiento de la actualidad y, finalmente, que recibieran una orientación en su búsqueda de información (Delgado y Oliver, 2009. Pág. 3). 
Con el objetivo de determinar la muestra que participaría en la proyecto, se seleccionaron durante los cursos 2010-2011 y 2011-2012 tres de los seis grupos en los que se engloban los alumnos y alumnas de primer curso de los tres grados del área de Comunicación que se imparten en la Universidad del País Vasco (Comunicación Audiovisual, Periodismo y Publicidad y Relaciones Públicas). Fruto de esta selección, sobre un universo total de 846 alumnos y alumnas matriculados durante los dos cursos académicos en los que se desarrolló el proyecto, la muestra quedó finalmente compuesta por 413 alumnos, lo que a nivel metodológico supone un nivel de confianza del $99 \%$ con un intervalo de 4.5 . De ellos un $49,9 \%$ pertenecía al grado de Periodismo, un 20,8\% al grado de Comunicación Audiovisual y un 29,3\% al grado de Publicidad y Relaciones Públicas.

\section{La plataforma gaurtest.info}

Entre las opciones existentes para el diseño y la publicación de los test de actualidad online, se optó por la plataforma virtual ProProfs (http://www.proprofs.com), una página web especializada en la elaboración de cuestionarios -entre ellos los de carácter académico- que permite numerosas posibilidades en la configuración de encuestas y de seguimiento de los datos recibidos (estadísticas, etc.). Por su parte, el medio elegido para proporcionar el contexto informativo a los cuestionarios fue la página informativa del ente público vasco eitb.com, dado que, además de abarcar todos los planos informativos - desde local a internacional-, realiza la misma cobertura informativa en euskera y castellano, un requisito indispensable para una actividad que involucra a grupos con docencia en ambos idiomas.

Una vez realizado el diseño de la propuesta, y con el inicio del periodo lectivo de la materia Géneros informativos durante el segundo cuatrimestre de cada curso, se ha publicado semanalmente un test de actualidad, al que podía accederse a través de la página web del proyecto, www.gaurtest.info. Los cuestionarios, en euskera o castellano según del grupo de docencia del que se tratara, han estado compuestos de 30 preguntas cada uno y han podido responderse de forma anónima, aunque se ha invitado a los estudiantes a que se identificaran para fomentar el máximo aprovechamiento de la actividad autoevaluadora y poder realizar un seguimiento de los resultados obtenidos y de su evolución a lo largo del cuatrimestre.

El enlace de cada pregunta a una noticia relacionada o a un video informativo ha permitido, además, ofrecer una contextualización de las informaciones, que dota a estas pruebas de actualidad de una doble función: quienes sólo han querido evaluar su competencia han podido responder las preguntas sin acceder a los contenidos relacionados; por el contrario, quienes además de medir su conocimiento de la actualidad han querido conocer más a fondo los temas sobre los que se pregunta, han podido visualizar los vídeos o consultar las noticias relacionadas. La plataforma, por tanto, 
ha permitido trabajar simultáneamente la autoevaluación de los conocimientos y el aprendizaje autónomo.

Imagen 1

Proyecto de Innovación Educativa 2010-2012.

Géneros informativos - Test de actualidad 14/05/2012

Question 6/30

Your Answer: Incorrect

¿Qué interés pagará la banca si pide ayuda para sanearse?

Si quieres más información sobre este tema consulta esta noticia.

A. Un $10 \% \vee$ (Correct Answer)

B. Un $2 \%$

C. Un $6 \%$

D. Un $18 \% \approx$ (Your Answer)

Next Question 》

Ejemplo de pregunta de actualidad en gaurtest.info.

En el diseño del cuestionario, cada una de las preguntas propuestas ofrece cuatro respuestas posibles, e indica en el momento de validar una de las opciones si ésta es la correcta o no (imagen 1). Con el objetivo de que el alumnado se concentre en la tarea autoevaluadora y evitar que la navegación en otros contenidos disperse su atención, el tiempo total para la realización de la prueba se ha limitado a diez minutos. Para ello, se ha procurado enlazar contenidos relacionados relativamente sintéticos, vídeos y noticias más bien breves.

Las pruebas han abarcado un amplio abanico de temas de actualidad general, englobados en seis grandes áreas (política, internacional, sociedad, economía, deportes y cultura), siempre con el propósito de reflejar la diversidad de la actualidad informativa. Esta distribución también ha buscado que el alumnado adquiera el hábito e interés por estar informado en todos los apartados de la actualidad, no sólo en los relacionados con sus intereses personales. Al finalizar la prueba, gaurtest.info señala el cómputo global de respuestas acertadas, y proporciona el listado completo de preguntas y respuestas con sus correspondientes vídeos/noticias para que el alumno pueda realizar una última comprobación o conservar un registro de la tarea realizada.

Desde el punto de vista formativo, la plataforma también ha permitido al profesorado de la materia Géneros informativos identificar las áreas en las que, de forma global, se perciben mayores carencias, lo que permite mejorar la orientación y asesoría que se realiza durante la docencia presencial en el aula.La identificación de los alumnos y las alumnas a la hora de realizar la prueba ha permitido, por su parte, reali- 
zar un seguimiento personalizado de su trabajo, detectar lagunas específicas y medir el grado de seguimiento no presencial de las tareas propuestas para que, más allá de la evaluación, puedan desarrollar unos hábitos e interiorizar unas rutinas.

Para reforzar la relevancia de esta competencia e incentivar el seguimiento de actualidad y la realización de los test, finalmente, la evaluación de la materia Géneros informativos, ha reservado un $10 \%$ de la nota final para este apartado.

\section{Resultado del aprendizaje}

Con el objetivo de completar los resultados obtenidos en los test de actualidad, y una vez finalizados los periodos de evaluación del segundo cuatrimestre de los cursos académicos 2010-2011 y 2011-2012, se recabaron las opiniones del alumnado participante sobre los resultados de aprendizaje de los test de actualidad online a través de encuestas voluntarias. Se recibieron un total de 196 respuestas, lo que supone un $47,46 \%$ de los participantes.

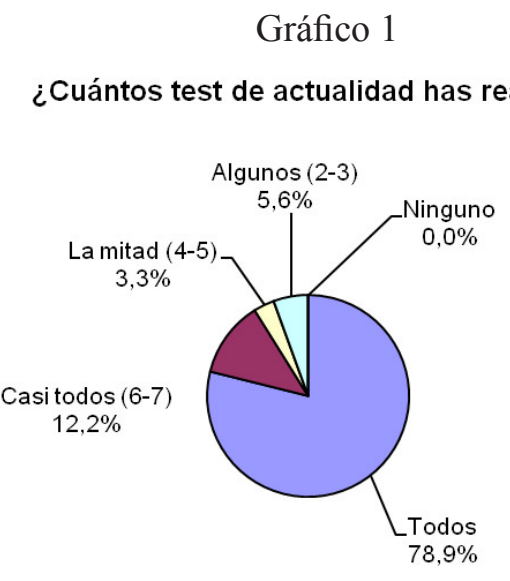

$\mathrm{N}^{o}$ de test realizados por alumno/a. Fuente: Elaboración propia

Las encuestas realizadas entre el alumnado revelan, en primer lugar, la alta aceptación y el uso intensivo que ha tenido la propuesta formativa. Un $91,1 \%$ de los encuestados (gráfico 1) afirma haber realizado todos o casi todos los ejercicios propuestos -6 ó 7-, una cifra muy destacable para una actividad voluntaria que se realiza principalmente en las horas de aprendizaje autónomo fuera del aula, en la que dos de cada tres test han sido realizados por los estudiantes desde su casa. 


\section{Gráfico 2}

\section{Tipo de medio de comunicación favorito para informarse (en \%)}

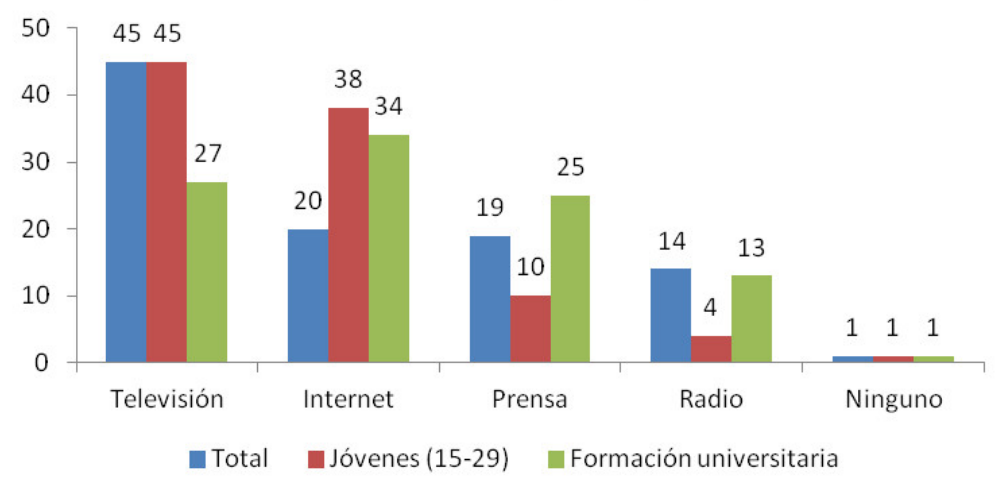

Tipo de medio favorito para informarse. Fuente: Ikuskerak 6

Estos datos, asimismo, se corresponden con fidelidad al número de test realizados contabilizados por la plataforma Proprofs -utilizada para diseñar la aplicación gaurtest.info- y que suman un total de casi tres mil a lo largo de los cursos 2010-2011 y 2011-2012, lo que arroja un promedio de 6,9 ejercicios realizados por cada alumno o alumna de los grupos participantes en este proyecto de innovación educativa, si bien estos datos incluyen a aquellos que han realizado el mismo ejercicio en más de una ocasión.

A la hora de responder sobre el medio de comunicación que preferían para informarse, en una escala del uno al cinco los medios de Internet lograron el primer puesto entre el alumnado, con una valoración media de 3,59 puntos, seguidos a corta distancia por la televisión y la prensa $-3,33$ y 3,31 respectivamente-, con la radio notablemente distanciada. Estos datos corroboran la preferencia de los jóvenes por los medios digitales, tal y como recogen los datos del sexto informe Ikuskerak (marzo de 2013) realizado por el Gabinete de Prospección Sociológica del Gobierno Vasco, que revela que en los colectivos de jóvenes (15-29) y de personas con formación universitaria la preferencia de informarse a través de Internet prácticamente duplica la media total (gráfico 3). 


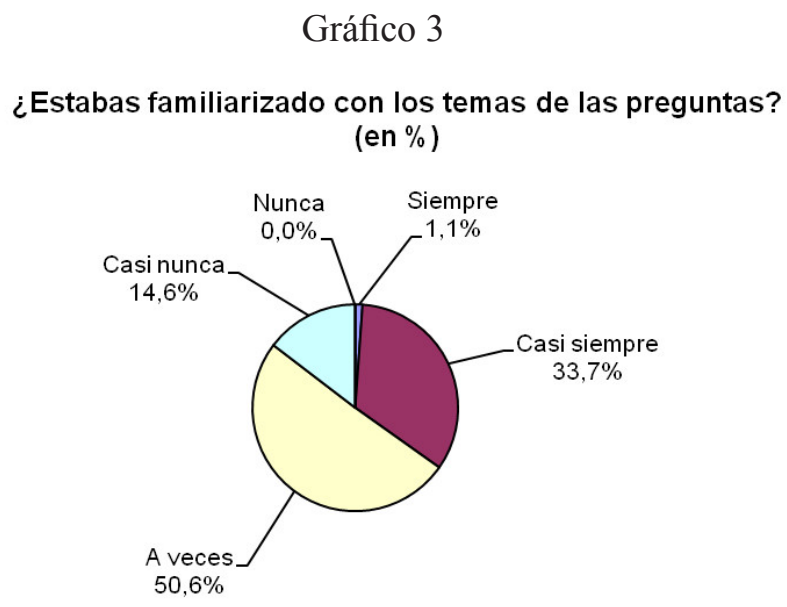

Conocimiento de los temas tratados. Fuente: Elaboración propia

Si la preferencia de los jóvenes por la información televisiva en detrimento de la radiofónica se ve confirmada, se aprecia por el contrario una significativa desviación en la alta aceptación que suscita la prensa escrita entre el alumnado que participó en el proyecto, que se aleja significativamente del magro $10 \%$ que la encuesta del Gobierno Vasco otorga a los jóvenes, y que bien puede atribuirse a que se trata de alumnos y alumnas de las carreras del área de la Comunicación, casi la mitad de los cuales están matriculados en el grado de Periodismo.

\section{Gráfico 4}

\section{Si no conocías la respuesta, ¿consultabas el contenido recomendado? (en \%)}

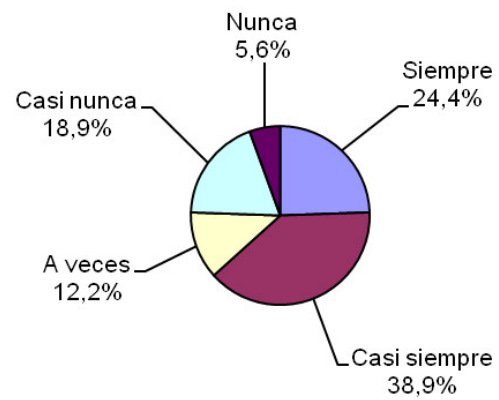

Consulta de contenidos recomendados. Fuente: Elaboración propia

La contextualización ofrecida en cada una de las preguntas también ha mostrado su utilidad como herramienta formativa. Frente al $34,8 \%$ del alumnado que afirmaba que 
estaba familiarizado con los temas informativos sobre los que versaban las preguntas 'siempre' o 'casi siempre', casi dos de cada tres de ellos señalaba que 'casi nunca' conocía esos contenidos, o que sólo le resultaban familiares 'a veces' (gráfico 4). Por el contrario, frente al $24,5 \%$ de los encuestados que afirmaban que no consultaban las informaciones enlazadas de eitb.com 'nunca' o 'casi nunca', el 63,3\% leía 'siempre' o 'casi siempre' dichas noticias. La herramienta de autoevaluación, por lo tanto, ha demostrado su capacidad para fomentar el hábito de informarse.

Con el objetivo de medir el grado de cumplimiento de los objetivos pedagógicos propuestos, se preguntó también al alumnado sobre el cambio en su hábito de consumo de información. Un $86,6 \%$ de los encuestados afirmaba seguir las noticias todos los días o varias veces por semana una vez finalizada la materia, frente al 64,3\% que lo hacía antes de que comenzara (gráfico 5). Este incremento de más de veinte puntos totales supone un significativo incremento en el consumo de información de actualidad y apunta a un perceptible cambio en los hábitos del alumnado.

Gráfico 5

¿Con qué asiduidad sigues las noticias? (en \%)

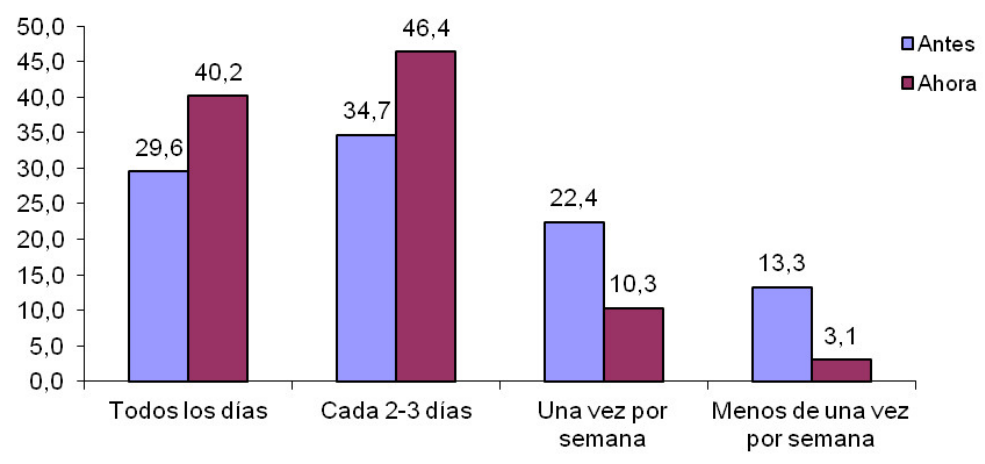

Evolución en el seguimiento de la actualidad informativa. Fuente: Elaboración propia

El cambio en la percepción subjetiva de los participantes sobre su grado de competencia en este campo resulta aún mayor. Si antes del comienzo de la materia Géneros informativos sólo un 19,6\% de los alumnos y alumnas consideraba que su conocimiento de la actualidad informativa podía calificarse como 'alto' o 'muy alto', esa cifra se elevó hasta un 50,6\% al finalizar el cuatrimestre. En el mismo periodo, el alumnado que consideraba que tenía un conocimiento 'bajo' o 'muy bajo' de la actualidad informativa descendió del 33\% a sólo el 4,2\% (gráfico 6). Una vez finalizado el periodo lectivo, en resumen, el alumnado que consideraba que tenía un nivel al menos 'medio' de esta competencia propia de la materia y de la titulación se elevaba por encima del $95 \%$. 


\section{Gráfico 6}

\section{¿Cómo valorarías tu conocimiento de la actualidad informativa? (en \%)}

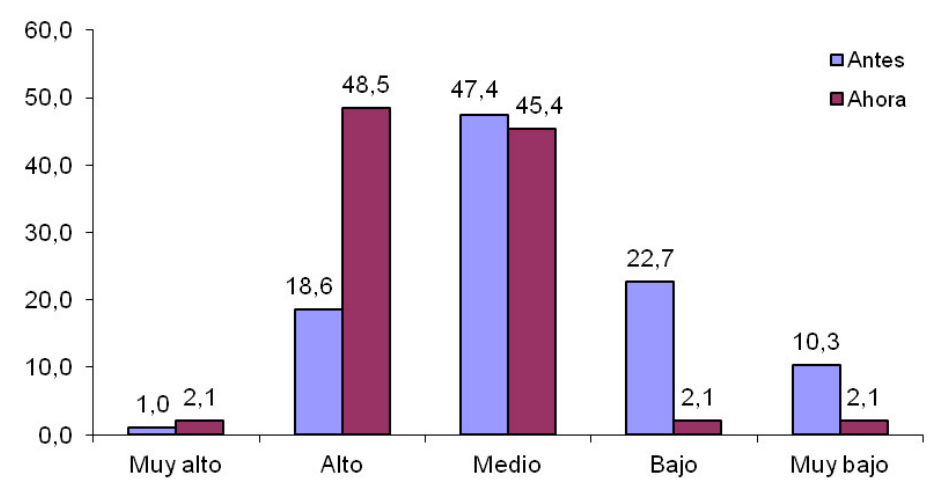

Valoración del conocimiento de la actualidad informativa. Fuente: Elaboración propia

Finalmente, la propia herramienta utilizada fue objeto análisis, y su capacidad para desarrollar la competencia deseada alcanzó entre el alumnado participante una valoración de 4,02 sobre 5 . Peor valorados fueron, por el contrario, el interés de los contenidos - con un 3,46- y su utilidad como herramienta de medición de la competencia requerida, $-3,5-$. En el primero de estos dos casos, la gran heterogeneidad de los temas sobre los que se preguntaba chocaba en muchas ocasiones con los propios intereses del alumnado, habitualmente concentrados en un número más limitado de temas. En el segundo caso, la relativa imprecisión de estos test de actualidad como herramienta para la medición de la propia competencia puede considerarse un déficit estructural, puesto que los contenidos de la actualidad informativa cambian de manera constante y, además, dos de cada tres participantes aseguraba que consultaba los contenidos complementarios que se le facilitaban, lo que obviamente desviaba muy al alza los resultados finales de aciertos obtenidos hasta superar el $75 \%$ de aciertos como promedio. La solución a este problema, en cualquier caso, la proporcionaba la propia aplicación, que permitía responder al test sin tener que consultar las referencias, y proporcionaba al final un sumario con todos los aciertos y errores, y los enlaces a todas las informaciones relacionadas. 


\section{Gráfico 7}

La plataforma Gaurtest (de 1 a 5)

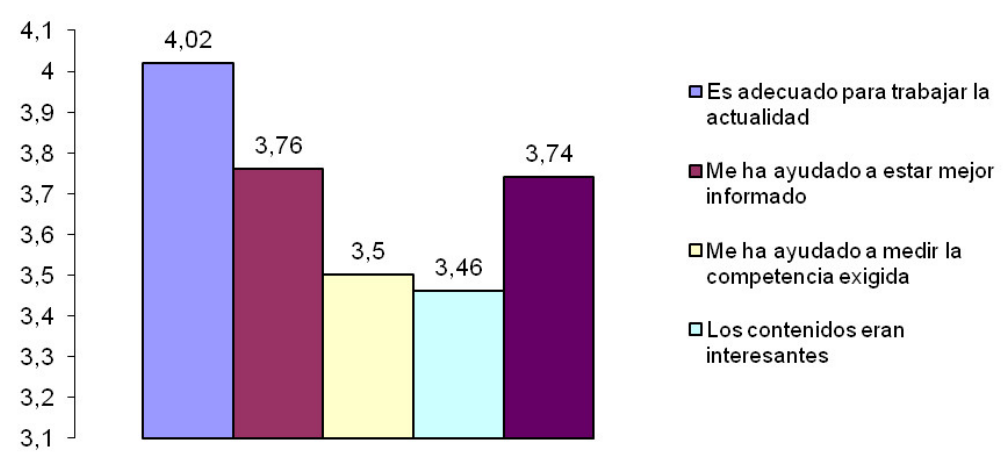

Valoración (1-5) de la plataforma gaurtest.info. Fuente: Elaboración propia

\section{Conclusiones}

El proyecto 'Los test de actualidad online como herramienta autoformativa para la adquisición de competencias en el área de comunicación', elaborado en el marco de la convocatoria de Proyectos de Innovación Educativa de la UPV/EHU, ha desarrollado la propuesta formativa gaurtest.info durante los cursos académicos 2010-2011 y 2011-2012, el análisis de cuyos resultados permite extraer las siguientes conclusiones:

1. La propuesta formativa de crear test de actualidad online ha tenido una gran aceptación como tarea de aprendizaje y autoevaluación propia de las horas de trabajo autónomo del alumnado. Los 413 alumnos y alumnas participantes realizaron un promedio de 6,9 test cada cuatrimestre, y un $91,1 \%$ completó todos o casi todos ellos, lo que demuestra su interés y utilidad pese a ser una actividad no presencial y voluntaria. Este alto índice de respuesta avala también la viabilidad y la eficacia de las herramientas online como complemento a la docencia presencial.

2. El seguimiento y la medición de la competencia de estar bien informado requerida en las titulaciones de grado del área de Comunicación demuestran la eficacia de los test de actualidad online. El número de alumnos y alumnas que siguen habitualmente los contenidos informativos de los medios de comunicación ha ascendido del $64,3 \%$ al $86,6 \%$ a lo largo del cuatrimestre en el que se ha impartido la asignatura Géneros informativos. La herramienta gaurtest. info también ha contribuido a que se incremente notablemente la percepción subjetiva del alumnado sobre su grado de competencia en el conocimiento de 
la actualidad, puesto que el número de alumnos y alumnas que se consideran debidamente informados ha pasado, por término medio, de un $67 \%$ antes de comenzar la materia, a más de un $95 \%$ después de finalizarla.

3. El uso de elementos contextualizadores en las pruebas de actualidad, favorecido por el uso de Internet como soporte, ha permitido asimismo reforzar el carácter formativo de propuesta mediante la asociación de las preguntas a las informaciones periodísticas que las responden. La elevada tasa global de acierto en los resultados del las pruebas, que asciende a un $77 \%$, sumada al hecho de que un $63,3 \%$ de los participantes afirmaba que habitualmente consultaba las informaciones recomendadas, deja de manifiesto que la plataforma gaurtest.info ha favorecido el hábito de leer y consultar las informaciones. En este apartado, la creciente preferencia de los jóvenes por los cibermedios en detrimento de los medios tradicionales, ha favorecido sin duda la buena acogida de la propuesta.

\section{Bibliografía}

BENITO JAEN, Á. (1995). La invención de la actualidad. Madrid: Fondo de la Actualidad Económica.

DE FONTCUBERTA, M. (1993). La noticia. Pistas para percibir el mundo. Barcelona: Paidós.

DELGADO GARCÍA, A.M.; OLIVER CUELLO, R. (2009) "Interacción entre la evaluación continua y la autoevaluación formativa: La potenciación del aprendizaje autónomo". En: Red-U. Revista de Docencia Universitaria. Accesible en: http://revistas.um.es/redu/article/view/ 92581/89071 [23-09-2013].

GARCÍA-BELTRÁN, Á.; MARTÍNEZ, R.; JAÉN, J.A.; TAPIA, S. (2006). "La autoevaluación como actividad docente en entornos virtuales de aprendizaje/enseñanza". En: RED. Revista de Educación a Distancia. Accesible en: http://www. um.es/ead/red/M6 [23-09-2013].

GOBIERNO VASCO. GABINETE DE PROSPECCIÓN SOCIOLÓGICA (2013). Ikuskerak 6. Vitoria-Gasteiz: Gobierno Vasco.

ITURREGUI, L.; AGIRRE, A.; LAZKANO, I. (2011). "Cómo formar en informarse: herramientas autoevaluadoras en materia de actualidad informativa para estudiantes de comunicación". En Actas del III Congreso Internacional Latina de Comunicación Social. Universidad de La Laguna. Accesible en: http://www.revistalatinacs.org/11SLCS/actas_2011_IIICILCS/180.pdf. [23-09-2013]

KAPUSCINSKI, R. (2003). Los cinco sentidos del periodista. México DF. FNPI.

PALOMARES, T. et al. (2005). "Las tecnologías de la información y la comunicación como factor de aprendizaje en la docencia universitaria". En GOÑI, Alfredo (ed.). Innovación educativa en la universidad. Bilbao. UPV/EHU.

PEÑA FERNÁNDEZ, S.; LAZKANO ARRILLAGA, I.; CANTALAPIEDRA GONZÁLEZ, M.J.; ITURREGUI MARDARAS, L.; AGIRRE MAIORA, A. 
(2011). "Herramientas autoformativas en el área de comunicación: Géneros informativos y los test de actualidad online". En PÉREZ DASILVA, J.A.; MESO AYERDI, K. y MENDIGUREN GALDOSPIN, T. Primeras Jornadas de Innovación Educativa y Nuevas Metodologías Docentes. Leioa. UPV/EHU, pp. 62-75.

\section{Los autores}

Simón Peña Fernández. Es doctor en Periodismo y profesor agregado en la UPV/ EHU. Sus principales líneas de investigación son el medios de comunicación en Internet, el diseño periodístico y la imagen del periodismo en el cine.

Iñaki Lazkano Arrillaga . Es doctor en Comunicación Audiovisual y profesor colaborador en la UPV EHU. Durante 15 años de trayectoria profesional en los medios de comunicación ha trabajado, entre otros, en los diarios 'Egunkaria', 'Berria' e 'Irutxuloko Hitza'. Es también colaborador habitual sobre materia cinematográfica en diversos medios.

Antxoka Agirre Maiora. Licenciado en Periodismo y en Derecho y profesor asociado en la UPV-EHU. Sus principales líneas de investigación son la interculturalidad a través de los medios de comunicación y el periodismo digital. Ha trabajado como periodista en diversas publicaciones culturales. 